\title{
TeV-PeV Cosmic-Ray Anisotropy as a Probe of the Local Interstellar Turbulence
}

\section{Gwenael Giacinti*}

Max-Planck-Institut für Kernphysik, Postfach 103980, 69029 Heidelberg, Germany

E-mail: giacinti@mpi-hd.mpg.de

\section{John G. Kirk}

Max-Planck-Institut für Kernphysik, Postfach 103980, 69029 Heidelberg, Germany

E-mail: john.kirk@mpi-hd.mpg.de

\begin{abstract}
We calculate the large-scale cosmic-ray (CR) anisotropies predicted for a range of GoldreichSridhar (GS) and isotropic models of interstellar turbulence, and compare them with IceTop data. In general, the predicted CR anisotropy is not a pure dipole; the cold spots reported at $400 \mathrm{TeV}$ and $2 \mathrm{PeV}$ are consistent with a GS model that contains a smooth deficit of parallel-propagating waves and a broad resonance function, though some other possibilities cannot, as yet, be ruled out. In particular, isotropic fast magnetosonic wave turbulence can match the observations at high energy, but cannot accommodate an energy dependence in the shape of the CR anisotropy. Our findings suggest that measurements of the large-scale CR anisotropy may be used as a local probe of the properties of the interstellar turbulence (notably its power-spectrum), and of CR transport, within a few tens of parsecs from Earth.
\end{abstract}

35th International Cosmic Ray Conference - ICRC2017

10-20 July, 2017

Bexco, Busan, Korea

\footnotetext{
* Speaker.
} 


\section{Introduction}

In this paper we report on investigations of how the shape of the large-scale (LS) anisotropy of $\mathrm{TeV}-\mathrm{PeV}$ cosmic-rays, (i.e., excluding features whose angular sizes are smaller than a few tens of degrees) depends on the properties of the interstellar turbulence and cosmic ray (CR) transport within $\sim 10$ pc from Earth [1]. Up until now, most studies of the large-scale CR anisotropy (CRA) have focussed on the direction and amplitude of the dipole, and in particular its relation to local sources of CRs (for a recent study see e.g. [2]). The direction of the CRA is observed to be compatible with that of the local interstellar magnetic field, as deduced from the IBEX ribbon and from polarization of starlight from stars within ten to a few tens of pc from Earth $[3,4,5]$. However, the LS CRA is not well described by a dipole [6]. A few earlier studies have modeled phenomenologically the LS CRA as the sum of a dipole and higher order multipoles (e.g. [7]), but the study we present here is, to our knowledge, the first quantitative description linking it to the power-spectrum and other parameters of the turbulence.

\section{Large-scale cosmic-ray anisotropy}

The alignment of the CRA with local field lines within $\sim 10 \mathrm{pc}[3]$ is compatible with the widely held view that CRs diffuse preferentially along field lines. Ref. [3] suggests that the power in the fluctuations on which TeV-PeV CRs scatter $\left(\sim 10^{-4}-1 \mathrm{pc} \ll 10 \mathrm{pc}\right)$ is small with respect to that in this ordered field. We therefore assume pitch-angle diffusion of CRs in a $\sim 10 \mathrm{pc}$-long, uniform flux tube containing the Earth. Assuming the turbulence to be homogeneous, and the problem to be $1 \mathrm{D}$ and stationary, the pitch-angle distribution of CRs $f(z, \mu)$ satisfies

$$
\mu v \frac{\partial f}{\partial z}=\frac{\partial}{\partial \mu}\left(D_{\mu \mu} \frac{\partial f}{\partial \mu}\right)
$$

where $z$ is the spatial coordinate along the tube, $\mu$ is the cosine of the CR pitch-angle (angle between the CR momentum and the ordered magnetic field), $D_{\mu \mu}$ the pitch-angle diffusion coefficient, and $v=c$. We place the Earth at $z=0$, set the tube boundaries at $z= \pm d \sim \pm 10 \mathrm{pc}$, and impose a non-zero CR flux along $z$. Around $z=0-$ cf. [8],

$$
f(z, \mu)=a_{\mathrm{diff}}[z+\tilde{g}(\mu)]
$$

provided that the boundaries are sufficiently far, i.e.

$$
\exp \left(-\Lambda_{1} d / v\right) \ll 1
$$

where $\Lambda_{1}$ is the smallest, non-zero, positive eigenvalue of (2.1), $a_{\text {diff }}$ a constant, and $\tilde{g}$ a solution of $\partial\left(D_{\mu \mu} \partial \tilde{g} / \partial \mu\right) / \partial \mu=v \mu$. See [1] for a delimitation of the parameter space where Eq. (2.3) is satisfied. Outside this range, $f$ is determined by the unknown boundary conditions - see [9] for an application to small scale anisotropies. In the following, we assume that (2.3) is satisfied. Then, the CRA at Earth is proportional to $\tilde{g}(\mu)$. Imposing $\int_{-1}^{1} \mathrm{~d} \mu \tilde{g}=0$,

$$
\tilde{g}(\mu)=-\frac{v}{2} \int_{0}^{\mu} \mathrm{d} \mu^{\prime} \frac{1-\mu^{\prime 2}}{D_{\mu^{\prime} \mu^{\prime}}},
$$


cf. [1]. Since we study here the shape of the CRA, and not its absolute amplitude, we work with the anisotropy normalized to 1 ,

$$
g(\mu)=\frac{\tilde{g}(\mu)}{\tilde{g}(1)} .
$$

$D_{\mu \mu}$ can be expressed in terms of a (phenomenological) resonance function, $R_{n}$ —see e.g. [10, 11]:

$$
D_{\mu \mu}=\Omega^{2}\left(1-\mu^{2}\right) \int \mathrm{d}^{3} k \sum_{n=-\infty}^{\infty}\left(\frac{n^{2} \mathrm{~J}_{n}^{2}(y)}{y^{2}} I_{\mathrm{A}}(\mathbf{k})+\frac{k_{\|}^{2} \mathrm{~J}_{n}^{\prime 2}(y)}{k^{2}} I_{\mathrm{S}, \mathrm{F}}(\mathbf{k})\right) \times R_{n},
$$

where $I_{\mathrm{A}, \mathrm{S}, \mathrm{F}}$ are the normalized energy spectra of the Alfvén (A), slow or pseudo-Alfvén (S), and fast (F) modes. $\Omega$ is the CR gyrofrequency, $y=k_{\perp} l \varepsilon \sqrt{1-\mu^{2}}, k_{\perp}$ is the component of the wavevector $\mathbf{k}$ perpendicular to the magnetic field, $l$ the outer scale of the turbulence, and $\varepsilon=v / l \Omega=r_{\mathrm{L}} / l$ the dimensionless $\mathrm{CR}$ rigidity. The physically unfounded case of isotropic pitch-angle scattering would correspond to a constant pitch-angle scattering frequency $v(\mu)=2 D_{\mu \mu} /\left(1-\mu^{2}\right) \times(l / v)=$ cst, and to a pure dipole anisotropy, $g(\mu)=\mu$.

We use broad $\left(R_{n}^{\mathrm{B}}\right)$ and narrow $\left(R_{n}^{\mathrm{N}}\right)$ resonance functions, taken respectively from [12] and [13]:

$$
\begin{gathered}
R_{n}^{\mathrm{B}}=\frac{\sqrt{\pi}}{\left|k_{\|}\right| v_{\perp} \delta M_{\mathrm{A}}^{1 / 2}} \exp \left(-\frac{\left(k_{\|} v_{\|}-\omega+n \Omega\right)^{2}}{k_{\|}^{2} v_{\perp}^{2} \delta M_{\mathrm{A}}}\right) \\
R_{n}^{\mathrm{N}}=\frac{\tau^{-1}}{\left(k_{\|} v_{\|}-\omega+n \Omega\right)^{2}+\tau^{-2}},
\end{gathered}
$$

where $v_{\|}=v \mu, v_{\perp}=v \sqrt{1-\mu^{2}}, \omega$ is the angular frequency of the waves, and $k_{\|}$the parallel component of $\mathbf{k}$. $R_{n}^{\mathrm{B}}$ takes into account the broadening of the resonance due to fluctuations of the magnetic field strength. This is encapsulated in the parameter $\delta M_{\mathrm{A}} \lesssim 1$, whose local value is poorly constrained. $R_{n}^{\mathrm{N}}$ assumes instead that the broadening of the resonance, described by $\tau$, is dominated by the Lagrangian correlation time of the turbulence.

\section{Results}

We use here models with $D_{\mu \mu}(-\mu)=D_{\mu \mu}(\mu)$. Thus, $v(-\mu)=v(\mu)$ and $g(-\mu)=-g(\mu)$, and we plot $v$ and $g$ on $0 \leq \mu \leq 1$ only. Typically, $l \sim(1-100)$ pc, so the dimensionless CR rigidity $\varepsilon=v / l \Omega$ is $\sim 10^{-4}-10^{-1}$ for $\sim \mathrm{PeV}$ CRs. We assume that the field points in the direction $(l, b)=\left(47^{\circ}, 25^{\circ}\right)$, which is compatible with [4].

\subsection{Goldreich-Sridhar turbulence}

First, we consider Goldreich-Sridhar (GS) turbulence [14], with a spectrum of Alfvén and pseudo-Alfvén waves following the prescription of [15]: $I_{\mathrm{A}, \mathrm{S}}(\mathbf{k}) \propto k_{\perp}^{-10 / 3} \exp \left(-k_{\|} l^{1 / 3} / k_{\perp}^{2 / 3}\right)$. In Ref. [1], we also studied spectra with an abrupt cutoff in $k_{\|}$and found qualitatively similar trends. The resonance function $R_{n}^{\mathrm{N}}$ provides so little scattering that the CRA is given by $g(\mu)$ only in a small fraction of parameter space, in which it anyway does not fit IceTop data. For GS turbulence, we then only use $R_{n}^{\mathrm{B}}$. We calculate numerically $D_{\mu \mu}$, and plot $v(\mu)$ (resp. the CRA, $g(\mu)$ ) in the upper left (resp. centre) panel of Fig. 1, for 3 sets of values of $\left\{\varepsilon, \delta M_{\mathrm{A}}\right\}$ that fit well IceTop 

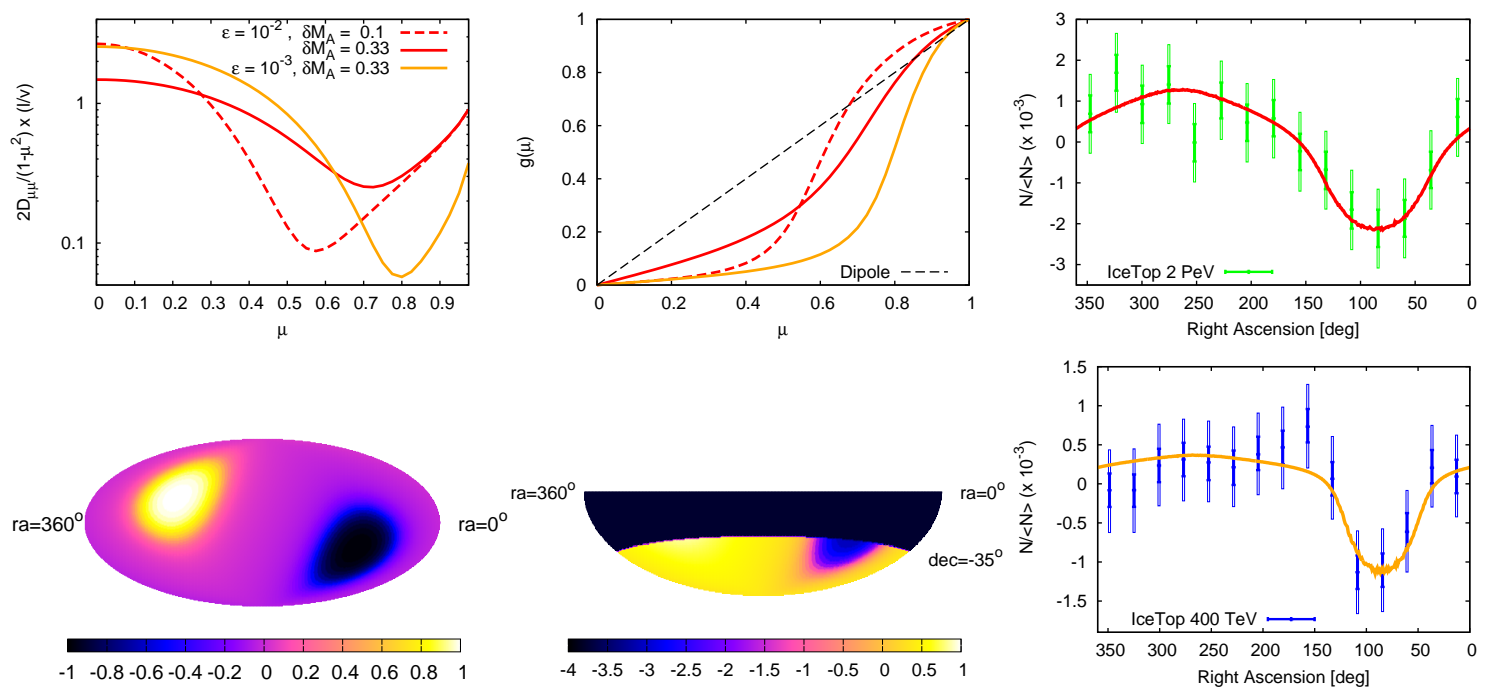

Figure 1: GS turbulence with $R_{n}^{\mathrm{B}}: v(\mu)$ (upper left panel), $g(\mu)$ (upper centre), and relative CR intensity at $-75^{\circ} \leq$ decl. $\leq-35^{\circ}$ versus R.A., compared with the $2 \mathrm{PeV}$ (resp. $400 \mathrm{TeV}$ ) data from IceTop (upper right, resp. lower right). See upper left panel for the parameters of each set of lines. Lower left panel: Anisotropy $g$ in equatorial coordinates for $\varepsilon=10^{-3}$ and $\delta M_{\mathrm{A}}=0.33$. Lower centre panel: CRA in the field of view of IceTop (calculated with respect to the average flux in each declination band) for the same $\varepsilon$ and $\delta M_{\mathrm{A}}$, and with minimum amplitude renormalized to -1 .

data [6]: $\left\{10^{-2}, 0.1\right\}$ (red dashed line), $\left\{10^{-2}, 0.33\right\}$ (red solid), $\left\{10^{-3}, 0.33\right\}$ (orange solid). For these parameters, $v$ exhibits a minimum in the range $|\mu| \simeq 0.55-0.8$ which corresponds to the transition between two regions: At smaller $|\mu|$ (i.e. perpendicular to field lines), scattering is dominated by the $n=0$ contribution of pseudo-Alfvén modes, whereas at larger $|\mu|$, Alfvén modes dominate. At fixed $\varepsilon$, and when $\delta M_{\mathrm{A}}$ increases, the minimum of $v$ occurs at larger $|\mu|$, cf. the two red lines. Indeed, the width of the bump around $\mu=0$ increases for broader resonances. The minimum of $v$ corresponds to the sharp increase of $g$ at large $\mu$, see centre panel and Eq. (2.4). This leads to excesses/deficits in the CRA along field lines $(\mu= \pm 1)$ that are narrower than for a dipole (black dashed line). We plot the anisotropy in (R.A., decl.) for $\left\{10^{-3}, 0.33\right\}$ in the lower left panel. Tight cold/hot spots are present along the field, and a rather wide flat region lies inbetween (magenta). This leads to a Southern sky map similar to what IceTop observes (lower centre panel): A narrow cold spot (dark blue) surrounded with a flat CR intensity. The two right panels show our predicted relative intensities at $-75^{\circ} \leq \mathrm{decl} . \leq-35^{\circ}$ versus IceTop data sets for a fixed set of turbulence parameters $\left(\delta M_{\mathrm{A}}=0.33\right)$ : At low CR energy $\left(\varepsilon=10^{-3}\right)$, we can fit well the $400 \mathrm{TeV}$ data, and by increasing the energy by a factor $10\left(\varepsilon=10^{-2}\right)$ - comparable to the factor 5 in the data, the $2 \mathrm{PeV}$ data is also well fitted. We note that the existing data can also exclude a non-negligible range of possible parameter values, see [1] for a wider scan.

\subsection{Isotropic Fast modes}

Second, we study isotropic fast mode turbulence with a power spectrum $I_{\mathrm{F}}(k) \propto k^{-3 / 2}$, as suggested by [16]. In Fig. 2, we plot $v$ (left), $g$ (centre), and the relative CR intensity at $-75^{\circ} \leq$ decl. $\leq-35^{\circ}$ versus IceTop $2 \mathrm{PeV}$ data (right). We consider $R_{n}^{\mathrm{B}}$ with $\delta M_{\mathrm{A}}=0.01$ (blue dashed- 

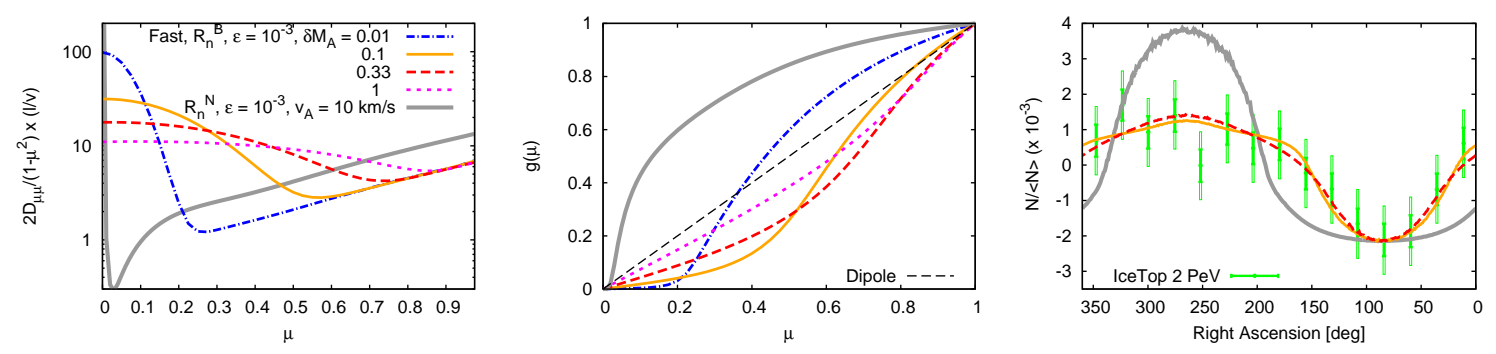

Figure 2: Isotropic fast modes: $v(\mu)$ (left panel), $g(\mu)$ (center panel), and relative $\mathrm{CR}$ intensity at $-75^{\circ} \leq$ decl. $\leq-35^{\circ}$ versus R.A., compared with the $2 \mathrm{PeV}$ data from IceTop (right panel). See left panel for the parameters of each set of lines.

dotted lines), 0.1 (orange solid), 0.33 (red dashed), 1 (magenta dotted), as well as $R_{n}^{\mathrm{N}}$ for a local Alfvén velocity $v_{\mathrm{A}}=10 \mathrm{~km} / \mathrm{s}$ (grey solid), and with $\tau=\sqrt{l} / v_{\mathrm{A}} \sqrt{k}$. We take $\varepsilon=10^{-3}$ for the plot of $v(\mu)$. We find that $g$ does not depend on $\varepsilon$ here. As for pseudo-Alfvén modes above, the $n=0$ term for fast modes is now responsible for the peak of $v$ around $\mu=0$. This peak grows in width when $\delta M_{\mathrm{A}}$ increases. With the narrow resonance function, it becomes very sharp and the minimum of $v$ is close to $\mu=0$, see grey line. This results in a qualitatively different shape of the CRA, cf. middle panel: Very wide cold/hot spots with a rather flat intensity inside. They take nearly half of the sky each $\left(\simeq 80^{\circ}\right.$ half-width). IceTop data clearly rules out this a priori acceptable scenario, cf. right panel. For $R_{n}^{\mathrm{B}}$, the $2 \mathrm{PeV}$ data is well fitted with $\delta M_{\mathrm{A}}=0.1$ and 0.33 , where the deficit at R.A. $\approx 90^{\circ}$ reaches its minimum width. However, the $400 \mathrm{TeV}$ data is not well fitted. Decreasing $\delta M_{\mathrm{A}}$ makes the deficit too wide (blue lines), while increasing $\delta M_{\mathrm{A}}$ to 1 (magenta lines) makes the anisotropy tend towards a dipole, which again increases the size of the deficit.

\section{Discussion}

In all models above, the CRA exhibits a flattening in directions perpendicular to field lines, due to the peak of the scattering frequency around $\mu=0$. Interestingly, this signature is compatible with the observational data. Moderately broad resonance functions are able to produce deficits/excesses along the field direction $(\mu= \pm 1)$ that are narrower than those of a dipole. Too broad a resonance would, however, result in an approximately dipole anisotropy with constant $v$, see the magenta lines in Fig. 2. On the other hand, narrow resonance functions are disfavoured by IceTop data. Nonetheless, we cannot formally exclude narrow resonance functions for models of turbulence in which $v$ is so small that the CR mean free path is $\gg 100 \mathrm{pc}$, such as in [13], since our study does not then apply. A very low CR scattering rate locally is not impossible, but shifts the problem of CR confinement and the explanation of its anisotropy to large distance. The increase with CR energy of the width of the deficit between the two IceTop data sets may hint at a $|\mathbf{k}|$-dependent anisotropy in the turbulence power-spectrum, such as in GS turbulence. Other possibilities, such as an energydependence of the resonance function should also be explored. As for fast modes, they should suffer from damping [12], which we did not take into account, and which may have an effect on the CRA. A combined analysis of all available CRA data (notably [17]) should yield tighter constraints than those presented in Sect. 3. Distortions of the CRA by heliospheric fields [18, 19] may complicate the picture at $\lesssim 10 \mathrm{TeV}$. The recent data from the Tibet Air Shower Array [20] 
hints at the presence of a narrow hot spot in the Northern hemisphere, in their $300 \mathrm{TeV}$ data set (cf. their Fig. 4), as would be expected within our model and for a $D_{\mu \mu}$ symmetric with respect to $\mu=0$.

\section{Conclusions}

Assuming pitch-angle diffusion of Galactic CRs in our local environment, we deduced the shape of the LS CRA, see Eqs. (2.4) and (2.5). In general, it is not a pure dipole, but contains imprints of the still poorly known properties of $(i)$ the local interstellar turbulent magnetic fields (e.g. power-spectrum), and (ii) CR transport (via $R_{n}$ ). We find that the existing observational data already puts constraints on these. A moderately broad resonance function seems to be favoured. IceTop $2 \mathrm{PeV}$ data can be fitted either with GS turbulence or isotropic fast modes (see parameters in Figs. 1 and 2), but only the former can reproduce the change in the width of the deficit between the $400 \mathrm{TeV}$ and $2 \mathrm{PeV}$ data sets. We suggest that the shape of the large-scale cosmic-ray anisotropy can be used as a new observable.

\section{References}

[1] G. Giacinti, J. G. Kirk, Large-Scale Cosmic-Ray Anisotropy as a Probe of Interstellar Turbulence, ApJ 835 (2017) 258 [arXiv:1610.06134].

[2] M. Ahlers, Deciphering the Dipole Anisotropy of Galactic Cosmic Rays, Physical Review Letters 117 (2016) 151103 [arXiv:1605.06446].

[3] N. A. Schwadron, F. C. Adams, E. R. Christian, P. Desiati, P. Frisch, H. O. Funsten, J. R. Jokipii, D. J. McComas, E. Moebius, G. P. Zank, Global Anisotropies in TeV Cosmic Rays Related to the Sun's Local Galactic Environment from IBEX, Science 343 (2014) 988-990.

[4] P. C. Frisch, B.-G. Andersson, A. Berdyugin, V. Piirola, R. DeMajistre, H. O. Funsten, A. M. Magalhaes, D. B. Seriacopi, D. J. McComas, N. A. Schwadron, J. D. Slavin, S. J. Wiktorowicz, The Interstellar Magnetic Field Close to the Sun. II., ApJ 760 (2012) 106 [arXiv:1206.1273].

[5] P. C. Frisch, A. Berdyugin, V. Piirola, A. M. Magalhaes, D. B. Seriacopi, S. J. Wiktorowicz, B.-G. Andersson, H. O. Funsten, D. J. McComas, N. A. Schwadron, J. D. Slavin, A. J. Hanson, C.-W. Fu, Charting the Interstellar Magnetic Field causing the Interstellar Boundary Explorer (IBEX) Ribbon of Energetic Neutral Atoms, ApJ 814 (2015) 112 [arXiv:1510.04679].

[6] M. G. Aartsen, R. Abbasi, Y. Abdou, M. Ackermann, J. Adams, J. A. Aguilar, M. Ahlers, D. Altmann, K. Andeen, J. Auffenberg, et al., Observation of Cosmic-Ray Anisotropy with the IceTop Air Shower Array, ApJ 765 (2013) 55 [arXiv:1210.5278].

[7] M. Zhang, P. Zuo, N. Pogorelov, Heliospheric Influence on the Anisotropy of TeV Cosmic Rays, ApJ 790 (2014) 5.

[8] N. J. Fisch, M. D. Kruskal, Separating variables in two-way diffusion equations, J. Math. Phys. 21 (1980) 740 .

[9] M. A. Malkov, P. H. Diamond, L. Drury, R. Z. Sagdeev, Probing Nearby Cosmic-ray Accelerators and Interstellar Medium Turbulence with MILAGRO Hot Spots, ApJ 721 (2010) 750.

[10] R. Kulsrud, W. P. Pearce, The Effect of Wave-Particle Interactions on the Propagation of Cosmic Rays, ApJ 156 (1969) 445. 
[11] H. J. Völk, Nonlinear Perturbation Theory for Cosmic Ray Propagation in Random Magnetic Fields, Ap\&SS 25 (1973) 471.

[12] H. Yan, A. Lazarian, Cosmic-Ray Propagation: Nonlinear Diffusion Parallel and Perpendicular to Mean Magnetic Field, ApJ 673 (2008) 942-953 [arXiv:0710.2617].

[13] B. D. G. Chandran, Scattering of Energetic Particles by Anisotropic Magnetohydrodynamic Turbulence with a Goldreich-Sridhar Power Spectrum, Physical Review Letters 85 (2000) 4656-4659 [arXiv:astro-ph/0008498].

[14] P. Goldreich, S. Sridhar, Toward a theory of interstellar turbulence. 2: Strong alfvenic turbulence, ApJ 438 (1995) 763-775.

[15] J. Cho, A. Lazarian, E. T. Vishniac, Simulations of Magnetohydrodynamic Turbulence in a Strongly Magnetized Medium, ApJ 564 (2002) 291-301 [arXiv:astro-ph/0105235].

[16] J. Cho, A. Lazarian, Compressible Sub-Alfvénic MHD Turbulence in Low- $\beta$ Plasmas, Physical Review Letters 88 (2002) 245001 [arXiv:astro-ph/0205282].

[17] M. G. Aartsen et al. [IceCube Collaboration], Anisotropy in Cosmic-ray Arrival Directions in the Southern Hemisphere Based on six Years of Data From the Icecube Detector, ApJ 826 (2016) 220 [arXiv:1603.01227].

[18] P. Desiati and A. Lazarian, Anisotropy of TeV Cosmic Rays and the Outer Heliospheric Boundaries, ApJ 762 (2013) 44 [arXiv:1111.3075].

[19] L. Drury, The problem of small angular scale structure in the cosmic ray anisotropy data, In Proceedings of the 33rd International Cosmic Ray Conference (Rio de Janeiro, Brazil, 2013) [arXiv:1305.6752].

[20] M. Amenomori, X. J. Bi, D. Chen, T. L. Chen, W. Y. Chen, S. W. Cui, Danzengluobu, L. K. Ding, C. F. Feng, Z. Feng et al., The Tibet AS $\gamma$ Collaboration, Northern Sky Galactic Cosmic Ray Anisotropy between 10 and 1000 TeV with the Tibet Air Shower Array, ApJ 836 (2017) 153 [arXiv:1701.07144]. 